\title{
ARTICLE \\ Orange Peel Extract Mediated Silver Nanofluid as Corrosion Inhibitor for X80 Steel in Simulated Oilfield Scale Dissolver
}

\author{
Ekemini Ituen* Chukwudurom Dim Ekerete BoEKOM
}

Department of Chemistry, Faculty of Science, University of Uyo, Uyo, Nigeria

\begin{tabular}{ll}
\hline ARTICLE INFO & ABSTRACT \\
\cline { 3 - 3 } $\begin{array}{l}\text { Article history } \\
\text { Received: } 25 \text { August } 2021\end{array}$ & $\begin{array}{l}\text { Silver nanofluid was prepared by bio-reduction reaction between orange } \\
\text { peels extracts (OPE) and silver nitrate and characterized by spectroscopic }\end{array}$ \\
Accepted: 2 September 2021 & $-50 \mathrm{~nm}$ and spherical shape were obtained. The nanofluid was applied \\
Published Online: 6 September 2021 & $\begin{array}{l}\text { as anticorrosion additive to inhibit corrosion of X8 steel in simulated } \\
\text { oilfield scale dissolver solution }(1.0 \mathrm{M} \mathrm{HCl}) \text { at various temperatures. }\end{array}$ \\
\hline Keywords: & The nanofluid (OPE-AgNPs) was $98.9 \%$ and $84.3 \%$ efficient at $30{ }^{\circ} \mathrm{C}$ \\
Adsorption & and 60 oC respectively as determined by weight loss measurement. In \\
Corrosion inhibitor & comparison with OPE, OPE-AgNPs shows better corrosion inhibition and \\
Orange peels extract & higher resistance to thermal degradation. Some kinetic and thermodynamic \\
Silver nanoparticles & models were used to characterize the inhibition process. OPE-AgNPs \\
Electron microscopy & could be optimized and used as alternative anticorrosion additive for scale \\
dissolution liquor in the industry.
\end{tabular}

\section{Introduction}

Scales are mineral deposits often accumulated downhole, on topsides or on export terminals during petroleum production. They plug the pipework or permeable reservior and cause flow problems. They also contaminate the produced fluid. Operators will be forced into relentless and costly effort to keep the pathway clear to maximize production. This is often achieved through descaling or acid wash operations to remove or decommission scales. The solution used for this procedure is often called scale dissolver and is composed mainly of hydrochloric acid of concentration about $1 \mathrm{M}^{[1,2]}$.

Being acidic in nature, scale dissolvers could create additional problems - corrosive attack on steel materials. Therefore, it is crucial to add corrosion inhibitors to the solution to retard the rate at which the dissolver corrodes the steel surface while maintaining the dissolver in the

desired activity. A wide variety of corrosion inhibitors (ranging from organic to inorganic, to organometallic compounds) are available for this purpose ${ }^{[3,4]}$. However, the corrosion inhibitor (CI) needs to be cheap and environmentally friendly, which makes plant extracts to attract tremendous research attention as potential material for use as corrosion inhibitor.

Plant extracts are cheap, readily available and nontoxic. However, plant extracts are easily degraded by heat and biochemical agents. In addition, it is difficult to store extract-based CIs for a long time as it will biodegrade ${ }^{[3]}$. Therefore, the need to chemically transform plant extracts into new materials that will not be easily degraded thermally or biochemically, and will exhibit improved inhibition efficiency and longer storage time becomes crucial. In this work, we report the efficiency of a nanofluid obtained by modification of plant biomass extract with silver nanoparticles as a new alternative

*Corresponding Author:

Ekemini Ituen,

Department of Chemistry, Faculty of Science, University of Uyo, Uyo, Nigeria;

Email: ekeminiituen@uniuyo.edu.ng 
corrosion inhibitor for X80 steel in simulated oilfield scale dissolver.

The advantage of nanoscale materials is that they have high size to volume ratio and this makes them more reactive. Nanoparticles prepared from plant extracts have been widely reported. Although the mechanism of formation of the nanoparticles has not been convincingly described, it is generally accepted to occur by bio-reduction reaction. Researchers opine that these nanomaterials have extensive future prospects. Better still, their synthetic process takes a single route and does not require complex tools or high energy. Since there are a wide variety of plants and the biomasses can be obtained in large quanttity, it is possible to scale up the production and have a wide range of nanoparticles for various applications. It is on these bases that our interest is channeled to this research direction.

\section{Materials and Method}

\subsection{Extraction}

Fresh ripe oranges were acquired from Itam market in Uyo and conveyed to Chemistry laboratory for analyses. The orange biomass was washed for two times in deionized water, peeled, air-dried, milled and weighed $\left(w_{0}\right) .10 .0 \mathrm{~g}$ of the pulverized biomass was extracted in 1.0 L deionized water for $24 \mathrm{~h}$ followed, then filtered. The residue was weighed $\left(w_{1}\right)$ after being dried in air. The weight of extract recovered was estimated by determining the difference in weight $\left(w_{0}-w_{1}\right)$. The extract solution was concentrated to paste form using rotary evaporator, then dried at $45^{\circ} \mathrm{C}$ to powder in the oven.

\subsection{Preparation and Characterization of Nanofluid}

$0.5 \mathrm{~g} / \mathrm{L}$ of the orange peels extract (OPE) was mixed with $1.0 \mathrm{mM} \mathrm{AgNO}_{3}(1: 1)$ at $30-50{ }^{\circ} \mathrm{C}$ in water bath with constant swirling until colour change was observed. A portion of the colloidal solution was dried to nano-powder in oven at $35{ }^{\circ} \mathrm{C}$ while the other portion was stored in amber bottle at laboratory temperature. A small amount of the nano-powder was dissolved in de-ionized water to obtain a colloidal solution of the nanofluid. The nanofluid were analysed for light absorption properties using UVVis spectroscopy at $400-800 \mathrm{~nm}$. Also, both the extract and nano-powder were characterized by X-ray diffraction (XRD) at $2=10^{\circ}-90^{\circ}$. Particle size, morphology and shape were also determined by TEM.

\subsection{Corrosion Studies}

1.0 $\mathrm{M} \mathrm{HCl}$ was prepared from 37\% Analar grade (Sigma
Aldrich) stock and used to simulate scale dissolver. X80 steel coupons of composition: (wt. \%) C (0.17), P (0.015), Ti (0.015), Si (0.38), S (0.002), Mn (1.25), Mo (0.20), Nb (0.04), Fe (bal), were used as test specimens in experiments. Specimens were initially immersed in absolute ethanol to remove lubricants, then polished with silicon carbide abrasives various grits until mirror finish was reached. Polished specimens were again cleaned in ethanol, washed in acetone for easy air-drying, then weighed.

Different weights of the nano-powder were measured and used to prepare three different concentrations $(0.10 \mathrm{~g} / \mathrm{L}, 0.30 \mathrm{~g} / \mathrm{L}$ and $0.50 \mathrm{~g} / \mathrm{L})$ in $1.0 \mathrm{M} \mathrm{HCl}$. The immersion approach was deployed to carry out weight loss experiments with the X80 steel coupons (dimension $=2 \mathrm{~cm} \times 2 \mathrm{~cm}$ ) fully suspended in the $1.0 \mathrm{M} \mathrm{HCl}$ (blank) and inhibited (containing the nano-powder) solutions at $30,40,50$ and $60{ }^{\circ} \mathrm{C}$ maintained in water bath ${ }^{[3]}$. After 24 $\mathrm{h}$ of immersion, each coupon was retrieved and cleaned following then ASTM standard procedures (ASTM G190) ${ }^{[5]}$, then reweighed. This was repeated for all test solutions in triplicates and the mean weight loss was determined which was used to computate corrosion rate and inhibition efficiency based on Equation 1 and 2 respectively.

$$
\begin{aligned}
& C_{R}=\frac{87.6 \Delta m}{\rho A t} \\
& \% I=100\left(\frac{C_{R O}-C_{R 1}}{C_{R O}}\right)
\end{aligned}
$$

where the parameters $\Delta m, A, t, \rho, C_{R O}$ and $C_{R 1}$ represent mean weight loss, surface area of coupon $\left(\mathrm{cm}^{2}\right)$, immersion time (h), density of iron $\left(\mathrm{gcm}^{-3}\right)$, and corrosion rates of blank and inhibited solutions, respectively.

\section{Results and Discussion}

\subsection{Formation and Characteristics of the Nanomaterial}

The nanoparticles were formed within 45 minutes as signalled by changing colour to dark brown. When allowed to stand for another $48 \mathrm{~h}$, the dark brown colour remained unchanged signifying that bio-reduction reaction had completed. Also, a homogeneous nanofluid without sediment was obtained which indicates that the nanopowder is soluble in water. Comparing the UV-Vis spectra of the extract solution and the nano-fluid, it was observed that there was difference in their plasmon absorption (Figure 1). While the extract absorbed at $\lambda_{\max }=545 \mathrm{~nm}$, the nanofluid absorbed at $\lambda_{\max }=496 \mathrm{~nm}$. The wavelength, $\lambda_{\text {max }}=496 \mathrm{~nm}$, falls within previously reported Plasmon 
absorption range of $400-500 \mathrm{~nm}{ }^{[6]}$ for plant biomass mediated silver nanoparticles (AgNPs), supporting that orange peels extract- silver nanoparticles (OPE-AgNPs) were completely developed. Absorption in the UV-Vis region often leads to electronic transitions. Depending on the identites of the phyto-compound, transitions such as $\sigma \rightarrow \sigma^{*}$ culd occur in single bonded molecules, $n \rightarrow \pi^{*}$ in molecules containing lone pair of electrons and $\pi \rightarrow \pi^{*}$ in double bonded, triple bonded and molecules with conjugation.

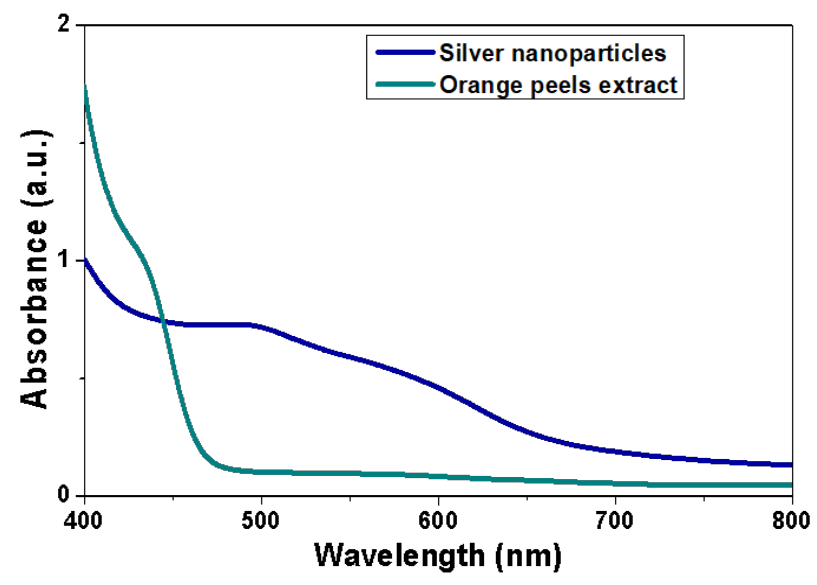

Figure 1. UV-Visible spectrum of orange peels extract and the silver nanofluid

To further investigate the nature of the nano-powder, XRD diffraction patterns of the pure extract and the OPEAgNPs were obtained (Figure 2). No well-resolved peaks were observed with the spectrum of OPE whereas distinct well-resolved peaks were observed for OPE-AgNPs at $2 \theta$ $=24.2,39.1,44.8,65.2,77.8$ and are indexed as (100), (111), (200), (220), and (222) on matching with JCPDS card ${ }^{[7]}$. It can be observed from the XRD spectrum of OPE-AgNPs that the peak corresponding to (111) plane has the highest intensity. This signifies that a large proportion of the nanoparticles exists in this orientation. Also, JPCD card matched the phase of silver to $\mathrm{Ag}^{0}$ indicating that the silver ions were actually reduced to zero valency which has been previously reported for other plant extracts mediated silver nanoparticles. Thus, the phytocompounds in orange peels extract act as reducing agent, reducing silver to zero valency while the silver ion acts as the oxidizing agent for the phytocompounds. With this scenario, the nanoparticles formed could be seen as resulting from capping of organic molecules of OPE on silver to form a coordinate covalently bonded complex which is organometallic in nature.

Average crystallite size $(\tau)$ of $62.6 \mathrm{~nm}$ was estimated from calculation using Scherrer's equation (Equation 3$)^{\left[{ }^{8]}\right.}$.

$$
\tau=\frac{k \lambda}{\beta \cos \theta}
$$

where $k$ is the shape factor, $\beta$ is the full with at half maximum (FWHM), $\lambda$ is the $\mathrm{X}$-ray wavelength and $\theta$ is the Bragg angle.

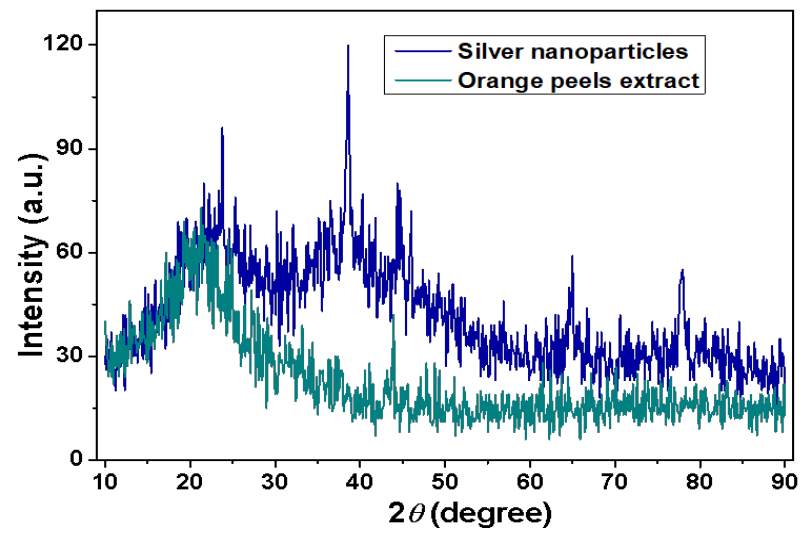

Figure 2. XRD diffraction pattern of Orange peels extract and the silver nano-powder.

The nanostructure, morphology and particle size of the nano-powder was determined by TEM and the image obtained is shown in Figure 3. It can be observed that nanoparticles were actually formed, and that the particles were almost uniformly round-shaped, non-agglomerated and around $55 \mathrm{~nm}$ in size. Particles with sizes range within $1-100 \mathrm{~nm}$ are usually considered to be nanoparticles ${ }^{[0]}$ and the average size of the synthesized material determined by TEM and XRD falls within this range.

\subsection{Corrosion Measurement}

The nano-powder was used as anticorrosion additive in the laboratory simulated scale dissolver liquor. The corrosion rate and inhibition efficiency obtained at different temperatures are presented in Table 1.

From the table, it can be observed that at all temperatures, corrosion rate was highest in the dissolver medium without the nano-powder. However, on addition of the nano-powder, corrosion rate reduces, indicating that corrosion has been inhibited. The inhibition efficiency increases as the concentration of the nano-powder increases but decreases on increase in temperature. The corrosion rate in the presence of the crude extract is higher than with the nano-powder, implying that the nanoparticles exhibit better anticorrosion effect than the crude extract.

At all temperatures and concentrations, the nanopowder yields higher inhibition efficiency than the extract implying that modification of the extract with silver nanoparticles improves its efficiency and resistance to 
Table 1. Corrosion rate and inhibition efficiency in the presence and absence of OPE and nano-powder (NP) at various temperatures for $24 \mathrm{~h}$ immersion time

\begin{tabular}{ccccccccc}
\hline \multirow{2}{*}{$\begin{array}{c}\text { Test } \\
\text { solution }\end{array}$} & \multicolumn{2}{c}{$30^{\circ} \mathrm{C}$} & \multicolumn{2}{c}{$40{ }^{\circ} \mathrm{C}$} & \multicolumn{2}{c}{$50{ }^{\circ} \mathrm{C}$} & \multicolumn{2}{c}{$60{ }^{\circ} \mathrm{C}$} \\
\cline { 2 - 8 } & $C_{R}(\mathrm{mmpy})$ & $\% I$ & $C_{R}(\mathrm{mmpy})$ & $\% I$ & $C_{R}(\mathrm{mmpy})$ & $\% I$ & $C_{R}(\mathrm{mmpy})$ & $\% I$ \\
\hline $1 \mathrm{M} \mathrm{HCl}$ & 0.3845 & - & 0.7239 & - & 1.5440 & - & 2.2714 & - \\
$0.5 \mathrm{~g} / \mathrm{L} \mathrm{OPE}$ & 0.0120 & 96.9 & 0.2992 & 58.7 & 0.7521 & 51.3 & 1.2102 & 46.7 \\
$0.10 \mathrm{~g} / \mathrm{L} \mathrm{NP}$ & 0.0058 & 98.5 & 0.1564 & 78.4 & 0.4625 & 70.0 & 0.7365 & 67.6 \\
$0.30 \mathrm{~g} / \mathrm{L} \mathrm{NP}$ & 0.0055 & 98.6 & 0.1164 & 83.9 & 0.3308 & 78.5 & 0.6098 & 73.2 \\
$0.50 \mathrm{~g} / \mathrm{L} \mathrm{NP}$ & 0.0043 & 98.9 & 0.0926 & 87.2 & 0.2305 & 85.1 & 0.4275 & 81.2 \\
\hline
\end{tabular}

thermal degradation ${ }^{[10]}$. This inhibitive effect can be attributed to the adsorption of the nanoparticles on the surface of the X80 steel causing a 'barrier' for mass and charge transfer thus protecting the metal surface from corrosion. Adsorption can be by physical or chemical mechanism. As obtained in this study, where inhibition efficiency decreases with temperature increase, it can be implied that previously adsorbed molecules may have desorbed as temperature increases. This behaviour is consistent with physical adsorption mechanism.

\subsection{Kinetic and Thermodynamic Studies}

Most bio-based CIs act by adsorption of their phytocompounds on active sites of the substrate (steel) ${ }^{[1]}$. This occurs by adherence of their molecules on the substrate surface and is often driven by chemical or physical interactive forces. The nature of the force could be predicted by fitting surface coverage into various adsorption isotherm models as a function of concentration. However, the concentrations in this study are not in molar terms, hence adsorption isotherms could not be determined. Instead, linear plots that fit Arrhenius equation (Equation 4) and thermodynamic transition state equation (Equation 5) were constructed as shown in Figure 3.

$$
\begin{aligned}
& \log C R=\log A-\frac{E_{Q}}{2.303 R T} \\
& \log \left(\frac{C R}{T}\right)=\left[\left(\log \left(\frac{R}{N h}\right)+\left(\frac{\Delta S^{*}}{2.303 R}\right)\right)\right]-\left(\frac{\Delta H^{*}}{2.303 R T}\right)
\end{aligned}
$$

where $C R$ is the corrosion rate, $N$ is Avogadro's number, $R$ is the universal gas constant, $h$ is Planck's constant, $A$ the Arrhenius frequency constant, $T$ is absolute temperature, $\Delta \mathrm{H}^{*}$ and $\Delta \mathrm{S} *$ is the enthalpy and entropy change of activation, respectively.

Relevant activation parameters (Table 2) such as the apparent activation energy $\left(\mathrm{E}_{\mathrm{a}}\right)$, enthalpy change $\left(\Delta H^{*}\right)$ and entropy change $\left(\Delta S^{*}\right)$ associated with the adsorption process were determined for all the test solutions. From results, $E_{a}$ values were higher when both OPE and NPs were added than in the free acid solution. In the presence of the NPs especially, higher $E_{\mathrm{a}}$ values were obtained. This indicates that the additives increase the energy threshold for ease of corrosion. Thus, their adsorption elicited corrosion resistance on the substrate surface.
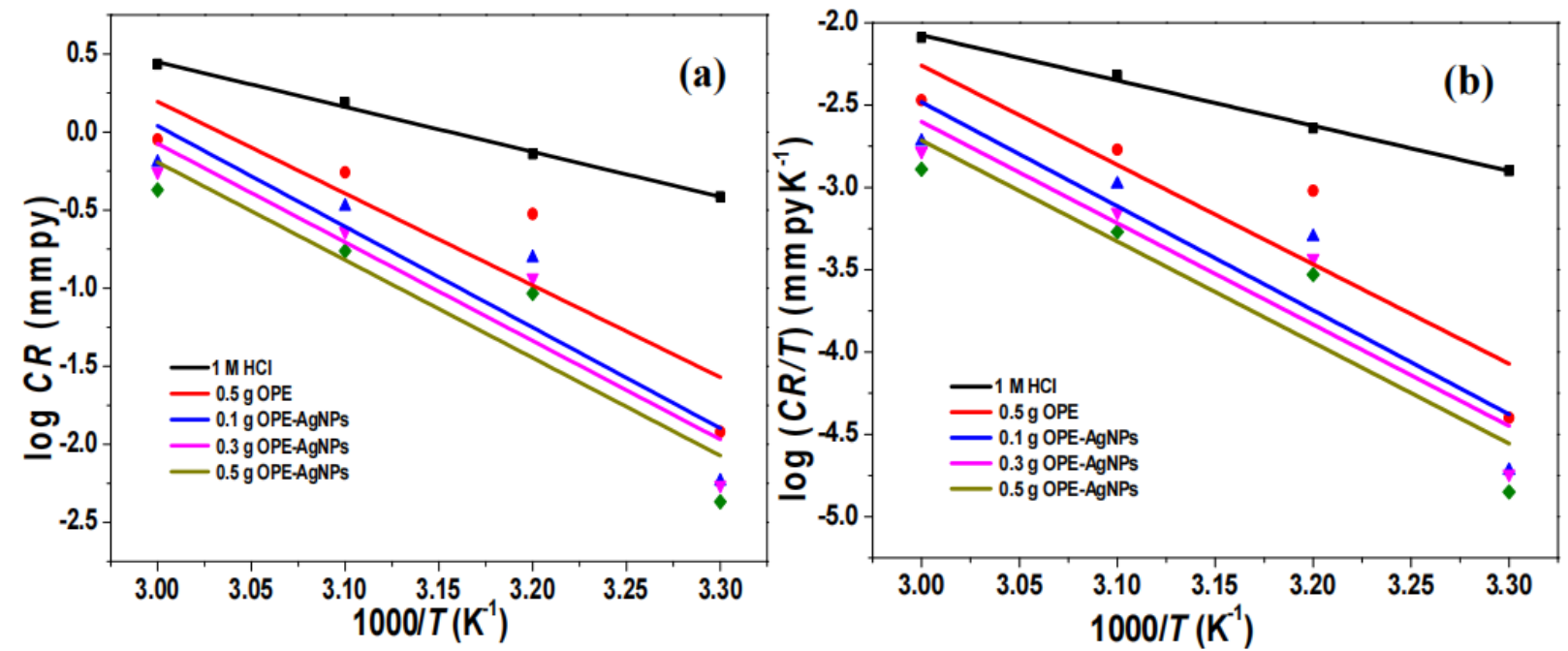

Figure 3. (a) Arrhenius plot and (b) Transition state plot for the corrosion of $\mathrm{X} 80$ steel in $1 \mathrm{M} \mathrm{HCl}$ containing OPE and the orange peel extract-silver nano-powders (OPE-AgNPs). 
This resistance increases as concentration increases, a performance that has been associated physical adsorption mechanism in literature ${ }^{[12]}$.

Table 2. Activation parameters obtained

\begin{tabular}{cccc}
\hline Test solution & $\boldsymbol{E}_{\boldsymbol{a}}(\mathbf{k J} / \mathbf{m o l})$ & $\boldsymbol{\Delta} \boldsymbol{H}^{*}(\mathbf{k J} / \mathbf{m o l})$ & $\Delta \boldsymbol{S}^{*}(\mathbf{k J} / \mathbf{m o l})$ \\
\hline $1 \mathrm{M} \mathrm{HCl}$ & 21.31 & -13.14 & 1.12 \\
$0.5 \mathrm{~g} / \mathrm{L} \mathrm{OPE}$ & 28.47 & -14.28 & 1.08 \\
$0.1 \mathrm{~g} / \mathrm{L} \mathrm{OPE}-\mathrm{AgNP}$ & 33.82 & -16.42 & 0.94 \\
$0.3 \mathrm{~g} / \mathrm{L} \mathrm{OPE}-\mathrm{AgNP}$ & 41.75 & -21.06 & 0.94 \\
$0.5 \mathrm{~g} / \mathrm{L}$ OPE-AgNP & 53.94 & -25.88 & 0.87 \\
\hline
\end{tabular}

Negative enthalpy changes were also obtained, which indicates that the adsorption was essentially exothermic, typical of physisorption mechanism ${ }^{[13]}$. The values of entropy change obtained were all positive and decreases in the presence of the additives in a concentration dependent manner. This indicates that the activated complex stage was dominated by association of molecules of inhibitor on the surface, rather than dissociation. Also, adsorption of the additives on the steel surface resulted in orderliness of the bulk solution ${ }^{[13]}$.

\section{Conclusions}

The following conclusions are drawn based on empirical data:

(1) Reaction between aqueous orange peels extract and silver nitrate produces nanofluid which can be dried to nano-powder.

(2) Orange peels extract-silver nanopowder are round shaped, of $55-63 \mathrm{~nm}$ average size and non agglomerating.

(3) The nano-powders inhibit corrosion of $\mathrm{X} 80$ steel in simulated scale dissolver at $30{ }^{\circ} \mathrm{C}$ to $60{ }^{\circ} \mathrm{C}$.

(4) OPE-AgNPs inhibits corrosion better than the crude extract especially at high temperatures.

\section{References}

[1] Rhudy, J. S. (1993). Removal of mineral scale from reservoir core by scale dissolver. In SPE International Symposium on Oilfield Chemistry. New Orleans, Louisiana, March 1993. SPE-25161-MS. https://doi. org/10.2118/25161-MS.

[2] Fink, J. (2021). Petroleum engineer's guide to oil field chemicals and fluids. Gulf Professional Publishing.

[3] Ituen, E., Akaranta, O. and James, A. (2016). Green anticorrosive oilfield chemicals from 5-hydroxytryptophan and synergistic additives for X80 steel surface protection in acidic well treatment fluids. Journal of Molecular Liquids, 224, 408-419.

[4] Cicek, V. and Ozdemir, M. (2013). Characterization
Studies Of Aqueous Immersion Solutions Of Novel Environmentally Friendly Organometallic Corrosion Inhibitors Used To Cure Mild Steel Substrates In Corrosive Media. International Journal of Engineering Research and Application 3(1), 1455-1461.

[5] Singh, A., Dayu, X., Ituen, E., Ansari, K., Quraishi, M. A., Kaya, S. and Lin, Y. (2020). Tobacco extracted from the discarded cigarettes as an inhibitor of copper and zinc corrosion in an ASTM standard D1141-98 (2013) artificial seawater solution. Journal of Materials Research and Technology, 9(3), 51615173.

[6] Ahmed, S., Ahmad, M., Swami, B. L. and Ikram, S. (2016). A review on plants extract mediated synthesis of silver nanoparticles for antimicrobial applications: a green expertise. Journal of advanced research, 7(1), 17-28.

[7] Priyaragini, S., Sathishkumar, S. R., \& Bhaskararao, K. V. (2013). Biosynthesis of silver nanoparticles using actinobacteria and evaluating its antimicrobial and cytotoxicity activity. International Journal of Pharmaceutical Science. 5(2), 709-712.

[8] Ituen, E., Yuanhua, L., Verma, C., Alfantazi, A., Akaranta, O. and Ebenso, E. E. (2021). Synthesis and characterization of walnut husk extract-silver nanocomposites for removal of heavy metals from petroleum wastewater and its consequences on pipework steel corrosion. Journal of Molecular Liquids, 335, 116132.

[9] Domingos, R. F., Baalousha, M. A., Ju-Nam, Y., Reid, M. M., Tufenkji, N., Lead, J. R., Leppard, G. G. and Wilkinson, K. J. (2009). Characterizing manufactured nanoparticles in the environment: multimethod determination of particle sizes. Environmental Science and Technology, 43(19), 7277-7284.

[10] Ituen, E., Singh, A., Yuanhua, L. and Akaranta, O. (2021). Green synthesis and anticorrosion effect of Allium cepa peels extract-silver nanoparticles composite in simulated oilfield pickling solution. SN Applied Sciences, 3(6), 1-17.

[11] Ituen, E., Akaranta, O. and James, A. (2017). Evaluation of performance of corrosion inhibitors using adsorption isotherm models: an overview. Chemical Science International Journal. 18(1), 1-34.

[12] Faraj, N., Abdelghani, K. A., Idress, A. A. and Ibrahim, D. M. (2021). L-Proline as a Green Corrosion Inhibitor in Aqueous Solutions for Carbon Steel. Journal of Metallic Material Research, 4(1), 16 -20.

[13] Chidiebere, M. A., Oguzie, E. E., Liu, L., Li, Y. and Wang, F. (2014). Corrosion inhibition of Q235 mild steel in $0.5 \mathrm{M} \mathrm{H} 2 \mathrm{SO} 4$ solution by phytic acid and synergistic iodide additives. Industrial and Engineering Chemistry Research, 53(18), 7670-7679. 